\title{
A la marge de la lecture et du ludique : les livres- jeux
}

\section{Boris Solinski}

\section{(2) OpenEdition}

1 Journals

Édition électronique

URL : http://journals.openedition.org/sdj/777

DOI : $10.4000 /$ sdj. 777

ISSN : 2269-2657

\section{Éditeur}

Laboratoire EXPERICE - Centre de Recherche Interuniversitaire Expérience Ressources Culturelles Education

\section{Référence électronique}

Boris Solinski, «A la marge de la lecture et du ludique : les livres-jeux », Sciences du jeu [En ligne], 7|

2017, mis en ligne le 20 février 2017, consulté le 28 mars 2021. URL : http://journals.openedition.org/ sdj/777 ; DOI : https://doi.org/10.4000/sdj.777

Ce document a été généré automatiquement le 28 mars 2021.

\section{(c) (i) (9)}

La revue Sciences du jeu est mise à disposition selon les termes de la Licence Creative Commons Attribution - Pas d'Utilisation Commerciale - Pas de Modification 4.0 International. 


\title{
A la marge de la lecture et du ludique : les livres-jeux
}

\author{
Boris Solinski
}

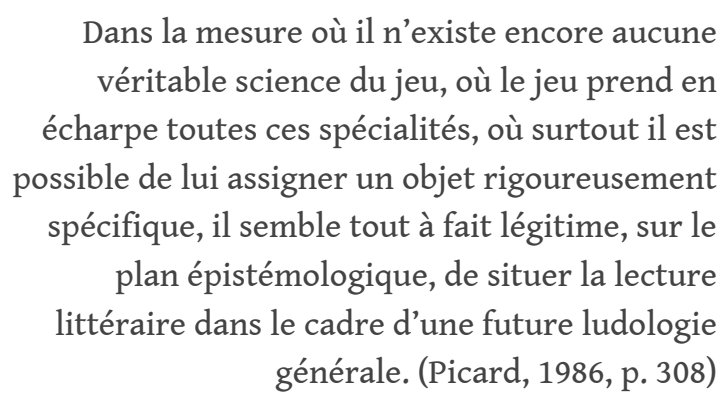

Si les études du jeu se sont en partie construites contre la narratologie, c'est sans doute parce qu'elles lui empruntent beaucoup, l'espace déployé par le terrain de jeu ne prenant corps qu'au fil du temps déroulé par la partie, espace et temps s'entremêlant dans l'expérience ludique. Alors que les théories de la lecture se revendiquent volontiers comme ludiques, la ludologie gagnerait à leur rendre la pareille. Car c'est à la frontière de la lecture et du jeu qu'il est possible de distinguer le moment ou l'une devient l'autre. De même que l'ethnologie a montré comment les peuplades qui vivent en marge du monde moderne peuvent nous en apprendre davantage sur nous-mêmes, de même les marges du jeu sont susceptibles de mettre en lumière la cristallisation des éléments ludiques qui lui donne sa fonction récréative. Pour ce faire, une piste possible est d'étudier à travers les livres-jeux les effets du ludique sur la narration et réciproquement - les effets secondaires de la première sur le second, ce que l'anglais nomme «effets de bord» (side effect). Car aucune forme littéraire ou ludique n'a aussi étroitement mêlé jeu et narration, la marge étant ici indissociable de leur (non) hybridation: à quel moment le livre-jeu bascule-t-il du livre vers le jeu, et réciproquement? Erving Goffman nous donne un indice :

Jouer, qu'il s'agisse de se battre ou de jouer aux dames, c'est, pour les participants, toujours jouer (...). Si la transformation systématique opérée par une modalisation n'altère que légèrement l'activité en question, elle modifie en revanche radicalement la définition qu'un participant pourra donner de ce qui se passe. On 
peut certes mettre en scène un combat ou une partie de dames, mais, pour les participants, la seule chose qui se passe réellement, c'est le jeu. La fonction cruciale d'une modalisation, c'est donc de définir ce qui pour nous est en train de se passer.

(Goffman, 1991, p. 54).

2 Par ce constat, le sociologue américain propose une explication à son modèle de la modalisation : le jeu n'est pas une forme qui peut être définie, mais une fonction («ce qui pour nous est en train de se passer ») qui peut prendre n'importe qu'elle forme. Il s'agit donc d'une approche ludologique puisqu'elle considère moins le jeu que le processus de compréhension qui nous fait le reconnaître comme tel, c'est-à-dire le ludique en tant qu'approche plutôt qu'objet d'étude, où la marge devient le lieu à même de dénouer la part de l'une, la ludologie, de celle de l'autre, la narratologie. Nous nous proposons ainsi d'aborder la lente maturation des livres-jeux de façon à la fois historique et fonctionnelle, à travers l'approche propre à chaque auteur en résonnance avec son époque.

\section{Du livre au jeu : l'approche narrative}

3 L'approche ludique est issue de celle littéraire qui, pour introduire un peu de jeu dans le modèle narratif, contrarie le paradigme littéraire où le lecteur tente d'anticiper ce qu'a préparé pour lui l'écrivain (Baroni, 2007). En effet, le livre-jeu innove en inversant la position de l'écrivain et du lecteur : l'écrivain essaie de prévoir les désirs du lecteur en lui proposant diverses alternatives, alors que ce dernier prend les rênes de la narration dont il est de fait le co-créateur puisqu'il en choisit le déroulement. La forme narrato-ludique reflète ainsi bien les contradictions de ses composants narratifs et ludiques dont Jorge Luis Borges pose les contraintes et les potentialités de leur hybridation en imaginant en 1941 un livre à l'image d'un labyrinthe, Le jardin aux sentiers qui bifurquent où peuvent être distinguées pour la première fois le livre et l'histoire qui en émerge :

Dans toutes les fictions, chaque fois que diverses possibilités se présentent, l'homme en adopte une et élimine les autres ; dans la fiction du presque inextricable Ts'ui Pên, il les adopte toutes simultanément. Il crée ainsi divers avenirs, divers temps qui prolifèrent aussi et bifurquent. (...) Cette trame de temps qui s'approchent, bifurquent, se coupent ou s'ignorent pendant des siècles, embrasse toutes les possibilités. De là les contradictions du roman. Fang, disons, détient un secret; un inconnu frappe à sa porte; Fang décide de le tuer. Naturellement, il y a plusieurs dénouements possibles: Fang peut tuer l'intrus, l'intrus peut tuer Fang, tous deux peuvent être saufs, tous deux peuvent mourir, et cætera. Dans l'ouvrage de Ts'ui Pên, tous les dénouements se produisent ; chacun est le point de départ d'autres bifurcations. Parfois, les sentiers de ce labyrinthe convergent. (Borges, 1983, p. 100-101)

4 Bien entendu, ce labyrinthe littéraire n'est pas détaillé par Borges qui se contente d'en évoquer les principes séduisants. Si un tel livre existait, c'est le lecteur qui devrait choisir entre les différentes alternatives et ses différentes potentialités afin de composer son expérience, en un mot ce livre serait illisible parce qu'il faudrait le jouer. Le labyrinthe est ainsi la métaphore des multiples possibilités qui convergent vers un cheminement unique, celui qui permet d'en sortir et qui n'est autre que l'expérience du lecteur qui le dénoue.

5 En 1967, Raymond Queneau, dans le cadre des expériences oulipiennes, propose une solution au problème imaginé par Borges en s'inspirant du jeu de l'Oie à travers 
l'histoire fantaisiste des «trois alertes petits pois ». Il existe un parcours meilleur que les autres qui ne sont qu'accidents, et les choix n'en sont pas vraiment puisque les alternatives qu'ils tranchent sont évidentes : «Désirez-vous connaître l'histoire?» «Préférez-vous une autre description?", le manque de coopération de la part du lecteur étant sanctionné à plus ou moins brève échéance par la fin du conte. Sans doute parce que, à l'instar de Borges que seule l'idée de la potentialité intéressait (« Un réseau croissant et vertigineux de temps divergents, convergents et parallèles. Cette trame de temps qui s'approchent, bifurquent, se coupent ou s'ignorent (...) embrassent toutes les possibilités.» Borges, 1983, p. 103), Raymond Queneau vise à représenter la potentialité, l'absurdité du propos ne faisant que souligner en creux la priorité donnée à l'innovation formelle à laquelle il se livre (figure 1).

Figure 1

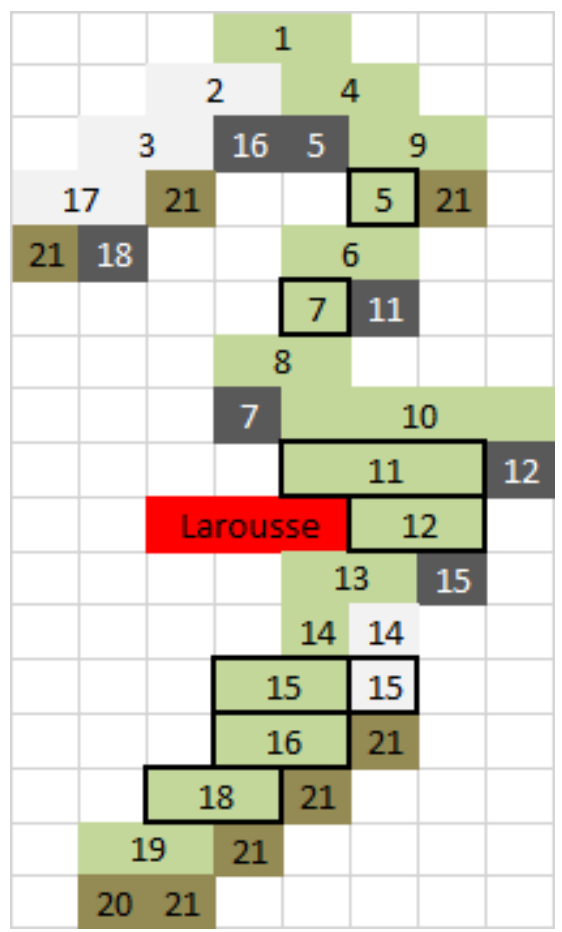

Arbre de possibilités d'Un conte à votre façon (Queneau, 1967 [notre représentation])

Les paragraphes numérotés montrent de haut en bas que les alternatives donnent rapidement lieu à des impasses (en noir) mettant fin à la narration, d'autant que l'histoire étant composée de 21 paragraphes, le renvoi au dernier d'entre eux met de toute évidence fin à la narration. Ainsi, à la façon du jeu de l'Oie, les conséquences de certaines étapes sont connues du lecteur avant même de prendre effet : « Si vous voulez savoir pourquoi ils s'éveillent d'horreur, consultez le Larousse au mot "ers" et n'en parlons plus» permettant au lecteur de les éviter afin de poursuivre sa lecture. L'objectif est pour Queneau de mener le lecteur jusqu'à l'extrême fin de l'histoire permettant à celui-ci de vérifier que le jeu n'est qu'un obstacle à la narration dont l'auteur retient, entre toutes les options qui s'ouvrent à lui, le développement le plus intéressant. Le jeu ne sert finalement que de caution à la narration.

7 Stimulé, son ami Georges Pérec décline l'année suivante cet ensemble de possibilités sous forme d'un organigramme d'une part et d'un texte de l'autre : L'art et la manière de 
demander une augmentation à son chef de service, texté adapté au théâtre en 1970 sous le titre L'augmentation. Si le lecteur ne peut plus infléchir le déroulement du récit, c'est bien la question de l'alternative qui justifie à elle seule le texte. Quel que soit le parcours retenu, l'organigramme ramène toujours à la situation initiale qui est celle d'avoir à demander une augmentation. Le texte, écrit sans ponctuation aucune afin de n'offrir au lecteur aucune possibilité de pause, est une logorrhée de 64 pages dont les alternatives empêchent la narration de progresser à la manière de digressions sans fin, celle-ci s'achevant sur la situation initiale où le lecteur est censé demander une augmentation. L'absurdité du propos vient de ce que l'ensemble des possibilités proposées au lecteur ne font que retarder le constat qu'aucune n'ouvre jamais sur celle d'obtenir une augmentation, le jeu se déroulant surtout à ses dépens.

Figure 2

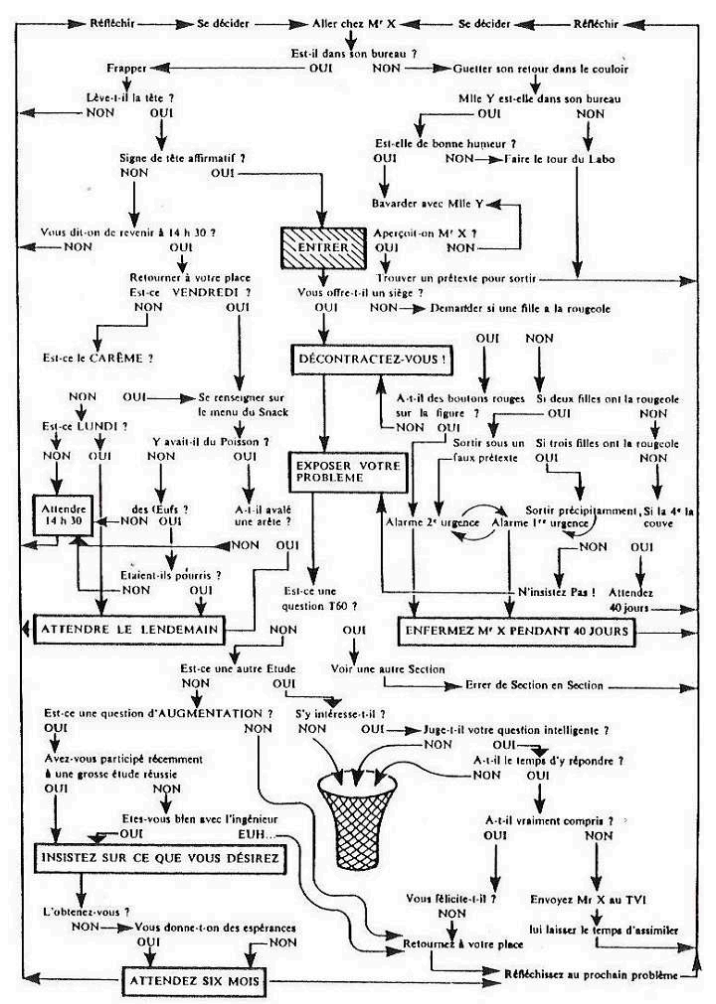

L'art et la manière d'aborder son chef de service exposés par organigramme (Pérec, 1968, p. 74-75)

Dès lors, le ludique vient contrarier la narration, l'étirant au point de lui ôter toute consistance par la multiplication de semblants d'alternatives, piégeant un lecteur condamné à échouer. Alors que chez Queneau le jeu soutient le principe même de la lecture en lui donnant sa signification, que ce soit dans Exercices de style (1947) ou Cent mille milliards de poèmes (1961), puisque sans lui celle-ci n'aurait aucun sens, chez Georges Perec la position est inverse, que ce soit dans La disparition (1968) ou Tentative d'épuisement d'un lieu parisien (1974) où le jeu est surtout celui de l'écrivain aux dépens du lecteur. Selon l'Oulipo, le jeu demeure avant tout une recherche conceptuelle à même d'ouvrir la littérature à de nouvelles possibilités créatives, mais dont l'intérêt tient essentiellement à l'originalité du procédé qui s'épuise de lui-même sans se féconder. 
Pourtant, de l'autre côté de l'Atlantique, l'Américain Edward Packard imagine en 1969, en s'appuyant sur le classique de la littérature jeunesse qu'est Robinson Crusoé, une histoire à même de se renouveler en faisant choisir au lecteur ses péripéties. Bien que publié seulement en 1976, Sugarcane Island fait d'un espace, "l'île canne à sucre ", le terrain de pérégrination du lecteur dont le parcours est à même de produire 38 fins différentes à partir d'un événement unique : l'arrivée du lecteur sur une île déserte.

Figure 3

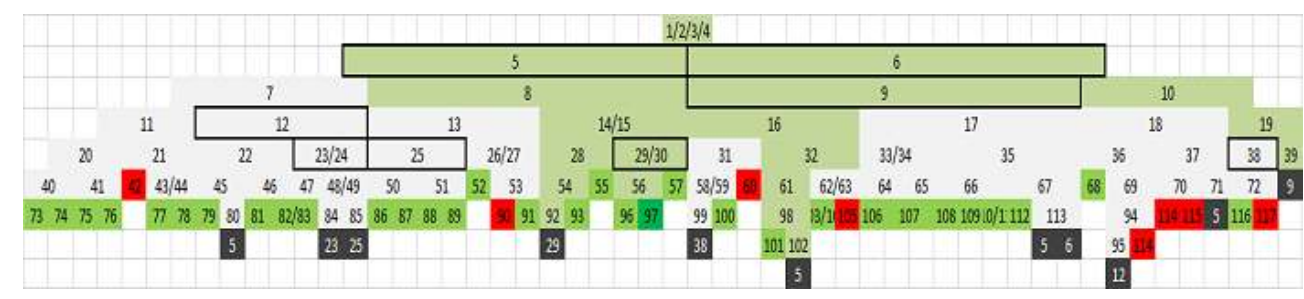

Arbre de possibilités de Sugarcane Island (Packard, 1976 [notre représentation])

L'arbre des possibilités, qui se déploie de haut en bas, montre une structure pyramidale où les numéros de paragraphes sont rangés par ordre croissant, de gauche à droite comme la lecture, et où la profondeur n'excède guère sept étapes. Cette représentation très éclatée montre une conception avant tout littéraire du jeu, où l'objectif reste de proposer le plus d'histoires différentes possibles à partir de la situation initiale qui conduit l'auteur à imaginer près de 40 fins. Au contraire du parcours proposé par Queneau, qui tente en permanence de faire converger les chemins de traverse pour alimenter l'histoire principale, Packard invente des histoires qui se croisent le moins possible. L'idée originelle est celle de réalités parallèles, comme en témoigne l'une des histoires qui fait de l'île un rêve (Packard, 1981, p. 76) alors que dans toutes les autres l'île est bien réelle. L'intention de l'auteur est donc moins de créer un monde, «l'île canne à sucre", qui réagit aux actions d'un joueur, que de proposer des variations autour d'un même thème. Avec un tel schéma, la narration la plus longue ne dépasse pas 20 étapes pour 103 possibilités, contre 17 étapes pour un total de 21 possibilités pour Queneau. D'autre part, l'unité littéraire pour son auteur reste la page alors que Queneau adoptait déjà le paragraphe, s'affranchissant davantage du support. Les problématiques pour ces deux auteurs sont ainsi contraires : s'amuser pour Queneau avec son lecteur en inventant un procédé de lecture original, l'histoire n'ayant qu'un intérêt secondaire, alors que pour Edward Packard il s'agit de multiplier les histoires possibles à partir d'un même point de départ.

11 Si les premiers essais de livres-jeux empruntent au jeu pour ouvrir de nouvelles pistes littéraires, le jeu demeure tout au plus un moyen quand l'objectif reste exclusivement littéraire. Borges ne règle pas les détails de son jeu, et qu'est-ce qu'un jeu sans règles ? Queneau fait avorter le principe même de choix au cœur du jeu en sanctionnant les mauvais choix par l'arrêt de la narration. Quant à Perec, il dénie tout enjeu à son jeu puisque les digressions ne sont pas de nouvelles potentialités de la narration mais son appauvrissement qui l'empêchent d'aboutir. Enfin, même le livre-jeu estampillé comme tel de Packard ne s'intéresse qu'à décliner le thème de l'île déserte au point de réduire la narration à sa plus simple expression, la diversité l'emportant sur la profondeur des choix nécessaires à l'expérience de jeu comme à celle de l'histoire. Le jeu, procédé pourtant au fondement du genre poétique, est ici traité comme un expédient (Borges) 
prétexte à une réflexion philosophique, ou comme un élément comique à même de désacraliser la dimension littéraire, par la dérision avec Queneau ou le grotesque avec Perec, à moins que ce jeu ne s'adresse explicitement à un public enfantin (Packard). Parce que la littérature honore ostensiblement le jeu en y recourant, elle montre qu'elle n'est pas encore prête à le reconnaître comme possibilité d'enrichissement, du moins au-delà de l'« exercice de style. ». A l'instar du mot-croisé, le jeu demeure en littérature un passe-temps, une récréation bien plus qu'une recréation.

\section{Du jeu au livre : l'approche ludique}

Il est logique que la forme canonique du livre-jeu ne soit pas le fait d'écrivains mais d'auteurs de jeux qui, dans une démarche plus populaire que littéraire, visent un public adolescent où le livre est à la fois alibi pour le jeu et prétexte à jouer. S'adressant d'abord à des joueurs, les auteurs et éditeurs de livres-jeux ont à cœur de les rendre accessible à un plus large public en permettant à ceux-ci de se passer du maître de jeu indispensable au jeu de rôle, mais aussi de règles complexes héritées des wargames, ou simplement parce que la "suspension de l'incrédulité " indispensable à la lecture fictionnelle s'accommode mal du retour au réel que provoque le lancer du dé1. Pour expliquer qu'il s'agit d'un jeu sous forme de livre sans le dire explicitement, les auteurs recourent à des comparaisons à la marge des jeux qui ont le mérite de souligner de façon explicite la mécanique ludique mise en œuvre. Si les comparaisons qui suivent avec les jeux de rôle et les jeux électroniques, et qui rencontrent un certain succès auprès des adolescents à la fin des années 70 , permettent de comprendre comment jouer seul (alors que par définition la lecture est un loisir solitaire), la comparaison avec l'enseignement programmé ${ }^{3}$ est censée expliquer l'impact du choix sur la lecture. Ces comparaisons audacieuses pour expliquer ce nouveau loisir au début des années 80 montrent dans tous les cas que la filiation que nous avons établie entre ces différentes formes ludiques et littéraires est loin d'être aussi évidente pour leurs auteurs, qui s'ignorent largement et ne peuvent tirer parti des expérimentations antérieures.

Il faut ainsi attendre 1982, soit l'année de la première édition du Conte à votre façon de Queneau, pour que, plus encore qu'un lecteur, un joueur soit placé au centre du procédé dans Le sorcier de la Montagne de feu. Premier opus des « Livres dont vous êtes le héros", cet ouvrage oppose à la multiplicité des expériences offertes par Sugarcane Island un enjeu unique. En effet, le but est de parvenir au paragraphe 400 qui représente la sortie du labyrinthe où le sorcier a caché son trésor, suggérant un parcours idéal parmi un ensemble de possibilités, mais aussi de multiples convergences entre les parcours potentiels (voir figure 4). La référence au jeu est immédiate à travers les jeux de rôle (eux-mêmes inspirés des wargames²) mais aussi les tentatives d'individualisation de l'enseignement programmé papier-crayon ${ }^{3}$ :

Conçu pour des aventures en solitaire, le livre fonctionne à la manière des jeux de rôle. Ceux-ci sont particuliers du fait même qu'un "meneur de jeu », qui préside en tant que dieu à l'aventure dans laquelle se lancent les joueurs, est indispensable. Dans Le sorcier de la Montagne de feu, le meneur de jeu est remplacé par le livre luimême, en usant d'un procédé familier à ceux qui ont suivi des cours d'enseignement programmé. (Jackson \& Livingstone, 1982, postface [notre traduction])

14 Il fait peu de doute que ni les jeux de rôle ni l'enseignement programmé ne sont pratiqués, au début des années 80 , par grand monde, a fortiori le grand public à même 
de s'intéresser aux livres jeux. Gallimard, l'éditeur français des «Livres dont vous êtes le héros », propose pour sa part en 1983 une comparaison avec les jeux vidéo : «Conçu pour un joueur solitaire, le livre fonctionne à la manière des jeux électroniques » (Jackson \& Livingstone, 1984, préface).

Le premier livre-jeu digne de ce nom tente alors une explication devenue depuis emblématique du genre :

Moitié roman, moitié jeu, ce livre est différent - un livre dont VOUS êtes le héros ! Armé de deux dés, d'un crayon et d'une gomme, lancez-vous dans une quête périlleuse pour découvrir le trésor du sorcier. VOUS aurez à décider quel itinéraire suivre et quels monstres affronter avec le système de combat élaboré présent dans le livre. Vous ne survivrez peut-être pas à votre première aventure. Mais avec l'expérience, l'habileté et la chance, chaque nouvelle tentative devrait vous rapprocher de votre but ultime... (Jackson \& Livingstone, 1982, $4^{\mathrm{e}}$ de couverture [notre traduction])

Figure 4

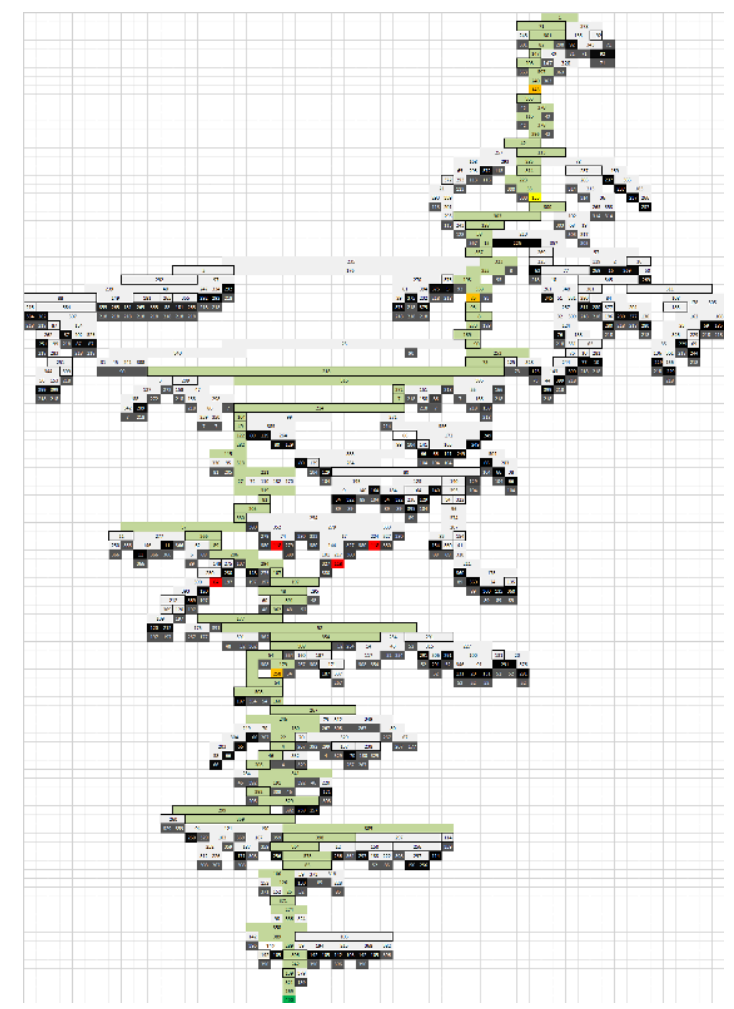

Arbre de possibilités du Sorcier de la Montagne de feu (Jackson \& Livingstone, 1982 [notre représentation])

En considérant le parcours le plus court en vert, l'arbre de possibilités du Sorcier de la montagne de feu montre une physionomie proche de celui d'Un conte à votre façon avec une trame principale et des digressions. Les cases noires, qui renvoient aux cases encadrées du parcours principal, permettent les convergences si peu présentes dans Sugarcane Island. Si les possibilités d'échec (en rouge) sont proportionnellement inférieures dans Le sorcier de la Montagne de feu, l'obligation de combattre les ennemis croisés pour gagner le droit de passer au paragraphe suivant nécessite une bonne gestion des ressources du personnage (points de vie, or, total d'endurance et de chance). D'autre part, les auteurs ont rajouté (en orange) des passages obligés pour 
l'obtention des clefs indispensables à l'ouverture du coffre du sorcier. Or les paragraphes où l'on trouve ces clefs agissent comme des verrous narratifs, obligeant le joueur à recommencer son exploration pour réussir enfin sa quête en évitant les impasses. Ce sont donc les choix du joueur qui conditionnent les péripéties ainsi que leur ordre, comme dans n'importe quel jeu, sauf que l'enjeu demeure toujours le même à la différence de Sugarcane Island qui, à l'inverse, raisonne narrativement:

Vous êtes confronté à des choix successifs et l'histoire dépend des décisions que vous prenez : vous pouvez être dévoré par un crocodile, découvrir un trésor caché, ou être secouru par un hélicoptère! (...) Que va-t-il se passer ensuite ? (...) l'aventure qui suit dépend de vous (...) Mais n'oubliez-pas! Réfléchissez avant d'agir - une erreur peut être votre dernière sur l'île canne à sucre ! (Packard, 1979, $4^{\mathrm{e}}$ de couverture [notre traduction])

Si le lecteur tourne les pages pour connaitre la suite, le joueur doit, pour sa part, faire preuve d'astuce pour surmonter les obstacles qui le séparent de l'enjeu. Ainsi, le lecteur de Sugarcane Island ne fait que choisir entre différentes histoires, représentées par 38 dénouements différents, positifs ou négatifs, alors que le joueur du Sorcier de la montagne de feu n'en connaît que deux : la réussite (incarnée par l'ultime paragraphe $\mathrm{n}^{\circ} 400$ ) ou l'échec, essentiellement en cas de défaite lors d'un combat.

A la différence d'un livre, qui met en scène les états d'âme et les motivations du héros, le livre-jeu abandonne cette dimension au lecteur puisqu'il ne peut rien connaître d'un héros qui n'est autre que son lecteur. D'autre part, un joueur a besoin de disposer des moyens de jouer, donc de choisir, que sont à minima l'enjeu et les conditions du choix. C'est pour cette raison que chaque paragraphe d'un livre-jeu rappelle précisément l'enjeu recherché et l'alternative que posent les voies pour y parvenir. Par exemple dans Sugarcane, l'enjeu : "Vous marchez sans fin à la recherche d'eau potable. Vous avez de plus en plus soif. » débouche sur le choix : "Si vous descendez vers le ruisseau, rendez-vous à la page 22 ; Si vous restez caché sur la corniche, rendez-vous à la page 23.» (Packard, 1978, p. 12). Le joueur est à même d'apprécier l'enjeu - sa survie - et les risques et bénéfices que comportent les deux options. Or, lorsque les choix ne concernent pas la conservation des moyens de jeu (le droit de poursuivre l'aventure), ils permettent alors la progression vers l'enjeu et le dénouement de la quête, comme par exemple ce choix arbitraire extrait du Sorcier de la montagne de feu (\$201) : «Vous avez le droit de ranger l'une de ces trois trouvailles dans votre sac à dos. » Il n'y a pas de raison impérieuse à cette obligation de choisir, sinon une occasion supplémentaire de solliciter la décision $\mathrm{du}$ joueur, tout en limitant sa progression vers l'enjeu. Cette influence de la mécanique ludique sur la narration montre que la cohérence de l'histoire est subordonnée à son intérêt ludique. C'est pour cette raison que le labyrinthe de la Montagne de feu, encore plus que la jungle de Sugarcane, déstructure le parcours de lecture en omettant les connexions entre ses éléments, faisant du parcours spatial du joueur, comme de son expérience temporelle, le lien de cohérence entre les lieux arpentés et les événements qui s'y produisent. Si le modèle de Sugarcane est logiquement à la fois une uchronie et une utopie du Robinson Crusoé de Daniel Defoe - où le tutoiement du premier fait écho au récit à la première personne du second -, le modèle du Livre dont vous êtes le héros est emprunté aux jeux de rôle et plus particulièrement au " porte - monstre - trésor $»^{4}$ que définit le standard de l'époque qu'est Dungeons \& dragons. Ainsi, isolée derrière sa porte, chaque salle peut receler un décor et une épreuve différant des précédents, seul le parcours du joueur leur donnant une cohérence. Le genre fantastique («heroic 
fantasy ") concourt en outre à limiter le réalisme à l'attrait historique d'un Moyen Âge de pacotille.

En prenant comme modèle le jeu de rôle, Les livres dont vous êtes le héros ont rétabli l'unité de la quête ludique qui correspond au format littéraire de la trame principale, améliorant la convergence des deux formes, narrative et ludique, avec le modèle du labyrinthe prôné par Borges, à la fois protéiforme et dont il n'existe pourtant qu'une sortie ou possibilité de réussite. A l'inverse, le cœur de l'aventure multiplie les possibilités de choix indissociables du risque d'échouer qui sont autant de défis tendus à la sagacité du joueur, renforçant sa fierté d'avoir surmonté toutes les épreuves sans trébucher. En réduisant la narration comme le jeu à leur plus simple expression, soit l'immersion fictionnelle et le choix, le livre-jeu souligne à la marge de ces formes culturelles proprement dites ce qui fait le fondement des approches narratives et ludiques. En effet, que l'on prenne en considération les définitions du jeu de Johann Huizinga (1951, p. 35) : «le jeu est une lutte pour quelque chose », de Roger Caillois (1958, p. 42-43) : « activité libre, séparée, incertaine, improductive, réglée, fictive. », de Jacques Henriot (1983, p. 38) « situation structurée par des règles, dans laquelle un sujet s'oblige à prendre un certain nombre de décisions", de Gilbert Boss $(2006$, p. 11) : " action réglée autonome ", ou encore de Colas Duflo (1997, p. 57) «l'invention d'une liberté dans et par une légalité ", ce n'est jamais que de choix dont il s'agit (Solinski, 2015, p. 198-203). Toute action n'est que l'expression d'une décision qui, en la réalisant, couronne le «bon " choix. Si Goffman s'intéresse au jeu afin de mettre au jour les valeurs des interactions (Goffman, 1974, p. 121 et sq), c'est que tout choix met en cohérence, au sein de l'expérience ludique, les alternatives réalisées (les réussites) avec celles qui ont été écartées (les épreuves ou risques).

\section{Représenter le ludique à partir des potentialités de choix offertes par les alternatives}

L'utilisation des arbres de Kuhn (schémas de possibilités) par la théorie des jeux permet d'embrasser d'un seul regard l'ensemble des choix offerts par les alternatives et, en ce qui nous concerne, celles écartées et renvoyées à la marge de l'expérience ludique, puisque le joueur juge sa performance pas tant par rapport à ce qu'il a réalisé qu'aux risques qu'il a surmontés, et par comparaison avec ce qui n'a pas eu lieu. Mais, alors que dans la théorie des jeux les choix représentés sont uniquement séquentiels et linéaires, les livres-jeux ont inventé une organisation circulaire de l'écriture qui, à l'image du labyrinthe de Borges, ouvre l'expérience de lecture, par la magie de l'alternative ludique, à la complexité des récursivités (retour à une alternative antérieure), rétroactions (changer les conséquences d'une alternative déjà tranchée), récapitulations (combinaisons d'alternatives) et autres ambivalences (possibilités de choisir plusieurs alternatives), en plus des raccourcis et des chemins de traverse. C'est moins la solution du livre-jeu (le tronc de l'arbre de possibilités) qui nous renseigne sur le ludique que les possibilités d'échecs, le ludique s'irréalisant dans les potentialités qu'il ouvre (les branches) plutôt que dans celles qu'il clôt. Nous nous proposons ainsi, à la suite du théoricien de la littérature Vladimir Propp (1970, p. 112-117), d'interpréter les fonctions du choix afin de sonder les profondeurs du ludique, autrement dit ses perspectives d'enrichissement qui prennent naissance dans les contraintes posées par les alternatives qu'il convient pour le joueur d'écarter afin de réussir. 
21 A la différence d'une démarche structuraliste, ce n'est pas la forme mais la fonction des choix qui nous intéresse et c'est à partir des embranchements que nous prétendons circonscrire celles-ci. Le concepteur de jeux Sid Meier fournit une explication sur laquelle s'appuyer :

Un jeu est une suite de choix intéressants. Dans un choix intéressant, aucune alternative n'est clairement meilleure que les autres, mais celles-ci ne sont pas uniformément attractives, et le joueur doit être à même de choisir en connaissance de cause (Rolling \& Morris, 1999, p. 38 [notre traduction]).

Une alternative intéressante suscite un choix difficile puisqu'elle fait hésiter entre plusieurs alternatives - plusieurs embranchements - possibles dont le joueur doit être en mesure d'apprécier les potentialités. Or si l'on considère, à la suite de Sid Meier, le ludique comme l'ensemble des potentialités ouvertes par le jeu, l'élaboration d'une typologie des choix offerts par le jeu permet la qualification ludique d'une situation donnée.

Cette typologie, appelée à être validée et complétée par l'expérience, permet de comprendre comment susciter la détermination du joueur peut stimuler son intérêt en lui soumettant une épreuve, celle de faire le bon choix. En effet, ce sont d'abord les contraintes ludiques (Duflo, 1997, p. 57 \& 80), les règles qui s'imposent de la même façon à tous, qui fondent l'intérêt du jeu grâce aux potentialités, ces fonctions que les contraintes ouvrent au joueur par l'obligation qui lui est faite de se dépasser pour réussir et étendre ainsi son propre potentiel, ce que Colas Duflo appelle la « légaliberté ». Le ludique n'est pas l'expression de la victoire mais seulement de sa possibilité, conditionnée tout autant par celle de la défaite. Toutes les fonctions des choix présentés ne sont pas exclusives mais cumulatives, permettant, par leur combinaison, de proposer au joueur un choix cornélien, pendant du paroxysme littéraire ou scénaristique. Ce n'est pas plus la multiplication dans un jeu de ces choix difficiles qui font son intérêt que celle des péripéties feraient celui d'une intrigue, mais bien la cohérence et la pertinence de leur combinaison au service de l'expérience, ludique ou narrative. De même, à l'instar d'une grammaire cinématographique, on en tirera difficilement des normes de design, mais plutôt un point de départ pour étayer une analyse. Les fonctions ludiques à même de rendre un choix difficile sont les suivantes :

\section{Le choix équitable}

Un choix équilibré est avant tout un choix équitable dont les alternatives proposent au joueur un bénéfice équivalent - sans être identique - à même de susciter l'hésitation. En général, il s'agit d'un équilibre entre les gains espérés et la difficulté de réaliser l'action, comme c'est le cas dans Le sorcier de la Montagne de feu (\$ 20): «Si vous êtes vainqueur rendez-vous au 376. Si le combat tourne mal vous pouvez fuir par la porte. Rendez-vous dans ce cas au 291 - mais n'oubliez pas votre pénalité de fuite.» Si le joueur préfère a priori remporter le combat, il doit éviter les blessures sous peine de compromettre la poursuite de son aventure. La possibilité d'éviter un affrontement en retirant seulement deux points d'endurance à son personnage peut s'avérer utile au joueur si celui-ci est affaibli, l'invitant à peser l'alternative de mener - ou ne pas mener - le combat à son terme. Visuellement une alternative équitable mène au même point mais par un chemin différent. 
Figure 5

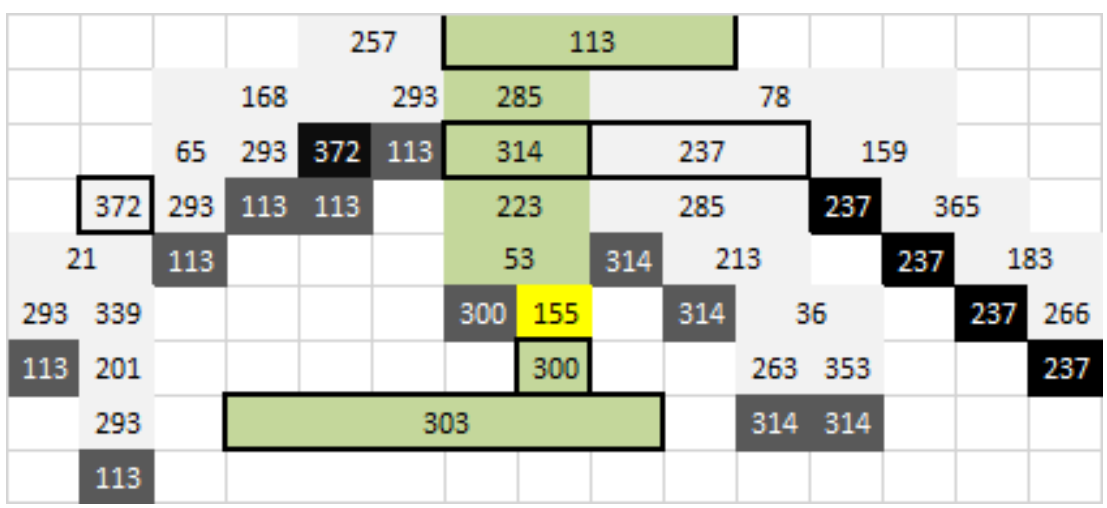

Un choix équitable dans Le sorcier de la montagne de feu (Jackson \& Livingstone, 1982 [notre représentation])

Depuis le haut du graphe de possibilités, le $\$ 113$ débouche ainsi sur deux parcours alternatifs, celui de gauche ramenant au point de départ (les cases grises et noires renvoient aux cases encadrées), celui de droite conduisant (après renvoi) deux paragraphes plus loin, au \$314, sans incidence ou presque sur la trame principale (en vert), sinon en apportant de la variété.

\section{Le choix risqué}

Un choix risqué comporte des contreparties explicites connues du joueur qui ajoutent de la tension à son choix, car il engage les choix réalisés jusqu'à présent mais aussi les conditions de poursuite de la partie, donc les chances de réussite du joueur. Ainsi, dans Le Sorcier de la montagne de feu (\$ 82): «Si vous essayez de voler la boîte, tentez votre Chance. Si vous êtes chanceux la créature ne se réveille pas - rendez-vous alors au 147. Si vous êtes malchanceux, rendez-vous au 33. " situe l'alternative dans le choix de subtiliser ou non le bien d'autrui, la prise de risque étant proportionnelle au bénéfice que le joueur en attend. Il s'agit d'un choix équilibré mais dont le risque anticipé est le paramètre déterminant de l'alternative.

Figure 6

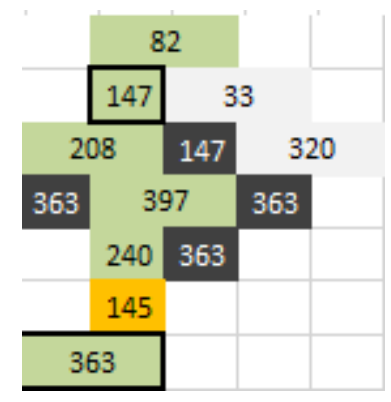

Un choix risqué dans Le sorcier de la montagne de feu (Jackson \& Livingstone, 1982 [notre représentation])

27 L'arbre de possibilités montre visuellement, de haut en bas, le risque que le joueur a de manquer le verrou scénaristique du $\$ 145$ (en orange) en empruntant à partir du $\$ 82$ le chemin secondaire (en gris) ou la trame principale (en vert) qui en conduisant directement au $\$ 363$ expose le joueur à ne pas trouver l'une des clefs indispensables à 
l'ouverture du coffre du sorcier. Il est à noter que, seul un joueur qui a déjà échoué une première fois peut être conscient du risque qu'il encourt, ce qui limite considérablement, dans l'exemple proposé, la fonction ludique du risque.

\section{Le choix ambivalent}

Un jeu est posé dès l'origine comme « Un système de règles définissant succès et échec, gain et perte » (Rey, 1994, p. 1070), c'est-à-dire par son ambivalence même. Si jouer c'est choisir, le propre d'une alternative est de proposer des options dont la réalisation empêche désormais celle des autres options, sans quoi il n'y a pas lieu de choisir entre pareil et même. L'image par excellence de l'ambivalence est celle du croisement, comme dans le labyrinthe de la montagne de feu: (\$128) «Vous pouvez maintenant aller jusqu'à la bifurcation. Choisissez-vous de poursuivre vers l'ouest (rendez-vous au 210), ou vers l'est (rendez-vous au 58) ? » Le fait de s'engager dans une direction éloigne irrémédiablement de l'autre, compromettant la possibilité de l'explorer ultérieurement au cours de la partie, invitant ainsi à rejouer. S'il ne s'agit que d'une image, toute règle est faite d'une contrainte et d'une potentialité obligeant à bien évaluer la part de l'une et de l'autre dans une alternative : s'engager et succomber ou renoncer et survivre, comme dans Ton nom de code est Jonas (Packard 1981b, p. 30) : «Suite page 100, si tu dis "naviguer à leur rencontre" ; page 102, si tu juges bon de prendre la fuite.», chaque option excluant toute possibilité d'explorer l'autre.

Figure 7

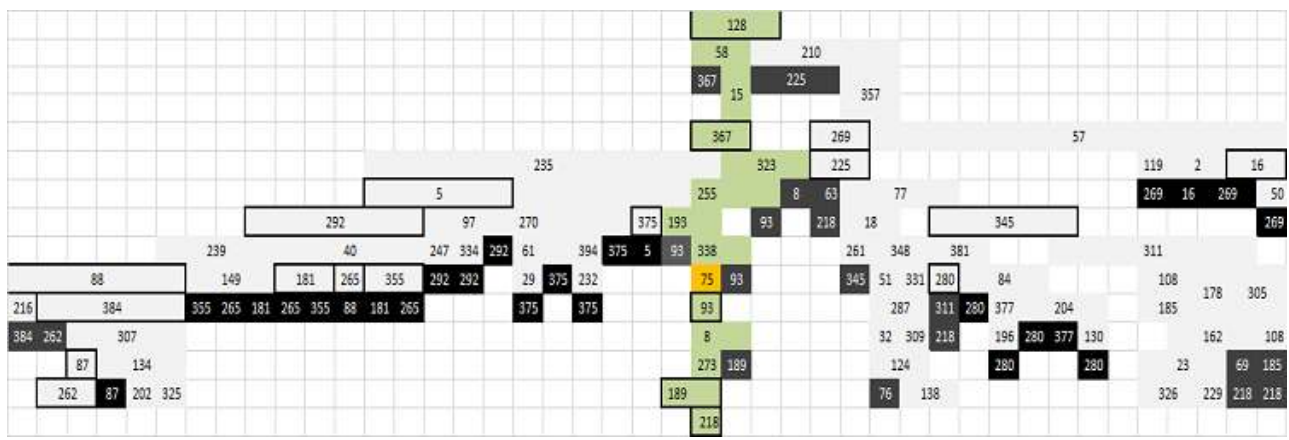

Un choix ambivalent dans Le sorcier de la montagne de feu (Jackson \& Livingstone, 1982 [notre représentation])

Dans cet arbre de possibilités, que l'on parcourt de haut en bas depuis le $\$ 128$, choisir l'option $\$ 210$ (en gris, à droite) ou, un peu plus bas, \$235 (en gris, à gauche) à partir du $\$ 367$ implique d'importantes disjonctions dans la trame qui différencient d'autant l'expérience ludique vécue par le joueur, le choix de l'une interdisant l'exploration de l'autre.

\section{Le choix décisif}

Le choix décisif est un choix susceptible d'entraîner la victoire ou la défaite immédiate. Dans le cas d'une issue potentiellement fatale, un indice est généralement fourni au joueur. Ce type d'alternative est plus courant dans les récits réalistes qui empruntent au Thriller, comme dans Ton nom de code est Jonas, où le joueur incarne un agent secret 
en pleine guerre froide qui tente de percer le mystère du langage des baleines plus rapidement que les Russes :

La Ford Noire se rapproche. Au croisement, la circulation reprend dès le changement de feu. Tu ne vois pas comment le chauffeur arriverait à passer, mais c'est peut-être votre seule chance. Suite page $46 \mathrm{si}$ tu dis au chauffeur de mettre l'accélérateur au plancher ; page 50 si tu lui dis de freiner. (Packard, 1979, p. 25)

Figure 8

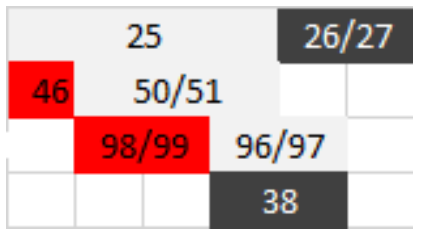

Un choix décisif dans Ton nom de code est Jonas (Packard ,1979 [notre représentation])

31 L'arbre des possibilités, qui se lit de haut en bas, montre que la page 25 renferme un choix décisif susceptible d'entraîner l'échec de la mission (en rouge), ou de mener à son tour à un autre choix décisif. La multiplication des impasses met ainsi le joueur sous tension en lui donnant l'illusion que sa vie est en jeu à chaque nouveau choix, le poussant systématiquement à peser le pour et contre tout en renforçant son immersion. On peut considérer qu'un choix décisif est un choix particulièrement risqué.

\section{Le choix incertain}

Le propre de l'alternative est de proposer une indétermination que la décision est à même de sonder en la tranchant. Si tout choix présente un degré d'incertitude, sans quoi il n'en serait pas un, dans un choix incertain le joueur méconnaît au moins un paramètre et le sait, la difficulté du choix découlant justement de l'impossibilité d'évaluer dans cette équation l'importance de l'inconnue. Or, à la différence d'une machine, lorsqu'un joueur choisit, il le fait en prenant en compte l'effort cognitif pour distinguer le choix optimal parmi l'ensemble des alternatives. Par exemple, dans $L a$ traversée infernale, le joueur doit se déterminer à partir d'une illustration, avec cette situation amusante que le personnage sait ce que le joueur ne connait pas encore (\$200) :

Pendant quelques instants, vous examinez attentivement le visage de vos compagnons de voyage et vous avez alors la certitude d'avoir deviné juste. Il ne vous reste plus qu'à passer à l'attaque. Mais qui est donc, selon vous, cet assassin présumé sur lequel vous aller vous précipiter à la seconde même ? (Dever, 1984) 
Figure 9

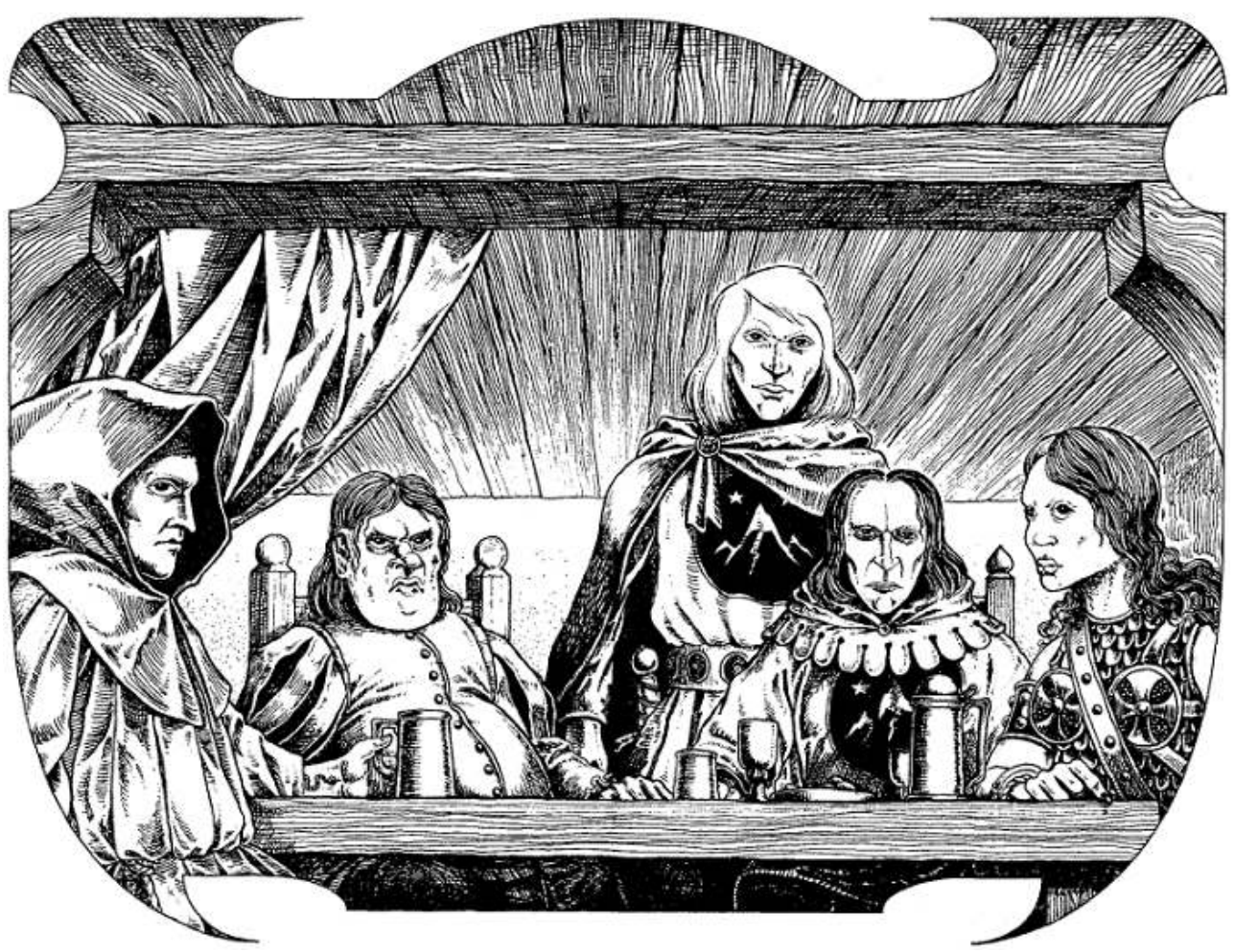

Un choix incertain dans le volume 2 de Loup solitaire (Dever, 1984, illustration de Gary Chalk)

Le joueur doit désigner l'une des cinq personnes de l'illustration comme son assaillant, soit au hasard en se rendant à l'un des paragraphes indiqués, soit en découvrant le détail qui lui donnera la même assurance que son personnage... Qu'il ait trouvé ou non, le joueur est à même d'évaluer le degré d'incertitude de sa réponse. De façon plus courante, dans les Livres dont vous êtes le héros, le choix incertain par excellence est celui du croisement : « Pour aller au nord, rendez-vous au 312 ; pour aller au sud, rendezvous au 246 ; pour aller à l'ouest, rendez-vous au 79 ; pour aller à l'est, rendez-vous au 349 (Jackson \& Livingstone, 1982) ».

Figure 10

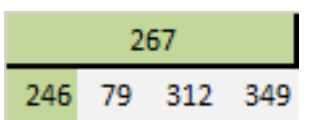

Un choix incertain dans Le sorcier de la montagne de feu (Jackson \& Livingstone, 1982 [notre représentation])

Dans cet arbre de possibilités, puisqu'aucun indice ne vient étayer le passage du \$267 aux quatre paragraphes inférieurs, le choix est fait soit au hasard, soit en fonction d'indices extérieurs à l'alternative elle-même : si le joueur griffonne une carte ou a déjà essayé au moins une autre direction. Mais dans tous les cas ce type de choix fait peser, faute de paramètres et d'enjeu pour se déterminer, un degré d'incertitude important quant à ses conséquences. On note ainsi que si tout choix requiert un minimum d'incertitude, se déterminer au hasard n'est plus choisir et n'a pas d'intérêt d'un point de vue ludique. 


\section{Le choix irrémédiable}

Est irrémédiable ce qui ne peut être annulé ou modifié. Les conséquences d'un choix peuvent généralement être infléchies par de nouveaux choix, dans la mesure du possible, mais le choix ludique a été effectué et il n'est pas possible de le reprendre. De même qu'un choix risqué, le choix irrémédiable implique l'évaluation de ce qualificatif au moment de se déterminer. Le cas le plus courant est un choix dont l'alternative ambivalente propose une option qui annule les autres, comme c'est le cas dans Le marais aux scorpions :

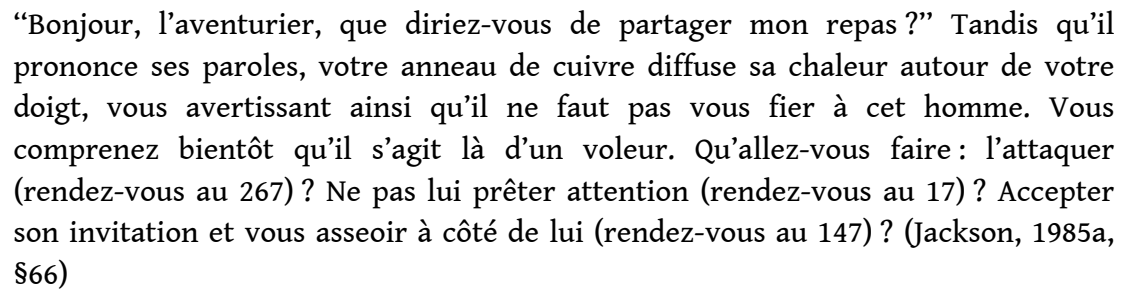

Si la première option empêche les deux autres, ces dernières vous exposent à vous faire dépouiller et à vous mettre dans l'impossibilité de défendre votre bien. Dès lors ces choix sont réciproquement irrémédiables (le choix de l'un exclut désormais le choix de l'autre). Un choix irrémédiable peut également avoir un caractère décisif quand il implique la fin de la partie, par exemple lorsque dans Ton nom de code est Jonas vous avez la possibilité de démissionner : "Suite page 90, si tu vas avec Hobbard mettre le président au courant; page 93, si tu donnes ta démission pour te consacrer désormais à la biologie marine. » (Packard 1981b, p. 88) En tant qu'espion, il est peu probable que la mission du joueur puisse se poursuivre une fois qu'il aura démissionné, ni qu'on lui offre une deuxième chance. Au lecteur donc d'apprécier les chances de réussite de cette option. 
Figure 11

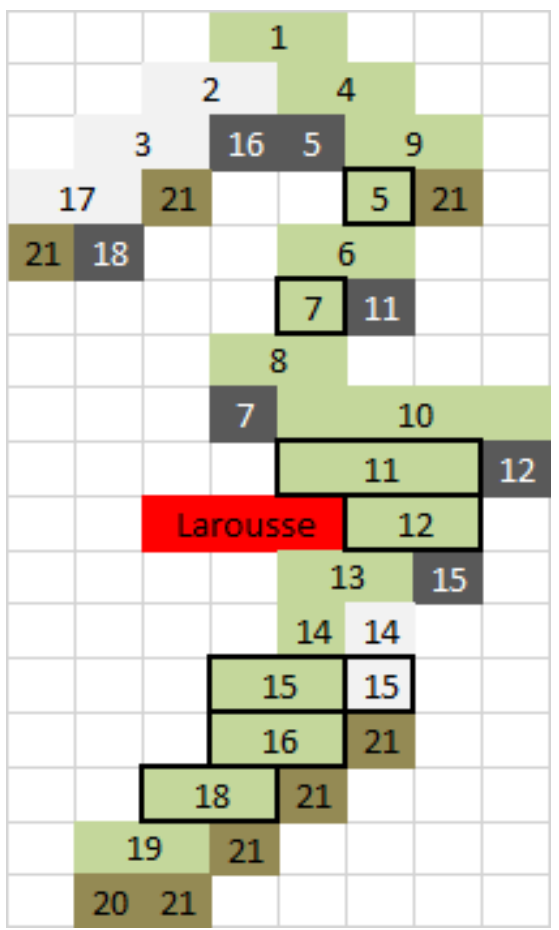

Un choix irrémédiable dans Un conte à votre façon (Queneau, 1967 [Notre représentation])

Dans ce graphe de possibilités, de haut en bas, on demande au lecteur $(\$ 1)$ : «Désirezvous connaître l'histoire des trois alertes petits pois ? - si oui, passez à 4. - si non, passez à 2. ». A partir du moment où celui-ci refuse (\$2 et suivants) il n’y a plus aucune possibilité de connaître l'histoire des trois petits pois, la narration prenant immédiatement fin ( $\$ 20 \& 21)$ ou presque.

\section{Le choix inévitable}

Un choix inévitable ne peut être repoussé, il est difficile soit parce que l'obligation de choisir ne laisse pas de délai pour se déterminer à loisir, soit parce qu'aucune alternative ne convient et que la seule option du joueur est de choisir la moins mauvaise. C'est le cas dans le premier Livre dont vous êtes le héros lorsque, quel que soit le choix effectué par le joueur, celui-ci est forcé, à un moment donné de son parcours, de se mesurer au sorcier de la montagne de feu :

Le combat va faire appel à toute votre force et à toute votre ruse. Votre adversaire s'est volatilisé, puis il a reparu à l'autre bout de la pièce devant une porte munie de deux serrures. Comment allez-vous l'affronter? (Jackson \& Livingstone, 1984, § 358) 
Figure 12

\begin{tabular}{|c|c|c|c|c|c|c|c|c|c|c|c|}
\hline & \multicolumn{4}{|c|}{274} & & & & & & & \\
\hline & & 98 & 356 & 324 & & & & & & & \\
\hline & \multicolumn{3}{|c|}{358} & & & & & & & & \\
\hline 142 & \multicolumn{3}{|c|}{389} & \multicolumn{8}{|c|}{105} \\
\hline 396 & \multicolumn{2}{|c|}{112} & 289 & 39 & \multicolumn{2}{|c|}{194} & \multicolumn{2}{|c|}{215} & \multicolumn{2}{|c|}{368} & 382 \\
\hline & 142 & 105 & \multicolumn{2}{|c|}{396} & 142 & 105 & 142 & 105 & 142 & 105 & 396 \\
\hline & 396 & & \multicolumn{2}{|c|}{242} & 396 & & 396 & & 396 & & \\
\hline & & & 139 & 379 & & & & & & & \\
\hline & & & 321 & 139 & & & & & & & \\
\hline & & & 169 & & & & & & & & \\
\hline & & & 400 & & & & & & & & \\
\hline
\end{tabular}

Un choix inévitable dans Le sorcier de la montagne de feu (Jackson \& Livingstone, 1982 [notre représentation])

En consultant l'arbre des possibilités de haut en bas, on voit que le $\$ 274$ présente trois options qui toutes mènent au $\$ 358$ qui obligent à combattre le sorcier, les autres options menant au $\$ 396 \ldots$ à condition bien entendu d'être vainqueur. Ainsi, arrivé au \$274, aucune autre alternative ne permet d'éviter la confrontation et son dénouement. Si sous cette forme le choix inévitable n'est guère qu'illusion de choix, renforçant seulement l'effort pour le repousser, son caractère inéluctable renforce la tension. Sous sa forme première d'obligation à choisir, un choix inévitable oblige à la prise de risque, celle de décider sans avoir pu peser le pour et le contre.

\section{Le choix intéressé}

Tout choix est intéressé sans quoi il ne servirait à rien de choisir. Cependant, la motivation du choix est pour beaucoup dans la tension qu'il suscite puisque la réussite ou l'échec sont alors plus lourds de conséquence. Le jeu intéressé par excellence est le poker, qui ne se joue pas de la même manière suivant qu'on mise des jetons ou de l'argent. Dans un livre-jeu, les conséquences ne sont jamais que fictives, et il est difficile de parler d'intéressement. Pourtant, dans Le marais aux scorpions, le joueur doit choisir un commanditaire avant de pouvoir explorer les marais :

Qu'allez-vous faire? Apporter votre aide à Gayolard le bon sorcier (rendez-vous au 335) ?; Prendre le risque de servir le mal en espérant toucher une bonne prime, et aller voir, dans ce cas, le sinistre Stratagus (rendez-vous au 255) ?; Essayer plutôt d'entrer en contact avec le mystérieux Pompatarte (rendez-vous au 27)? (Jackson, 1985a, §205)

41 Il est visible dans cette alternative que faire le bien se suffit à lui-même, alors que servir un maître neutre demande davantage de motivation et donc ici d'informations, et à fortiori que faire le mal exige une contrepartie pécuniaire. Bien que toutes ces récompenses soient fictives, le joueur a besoin de cette illusion pour se prendre au jeu. 
Figure 13

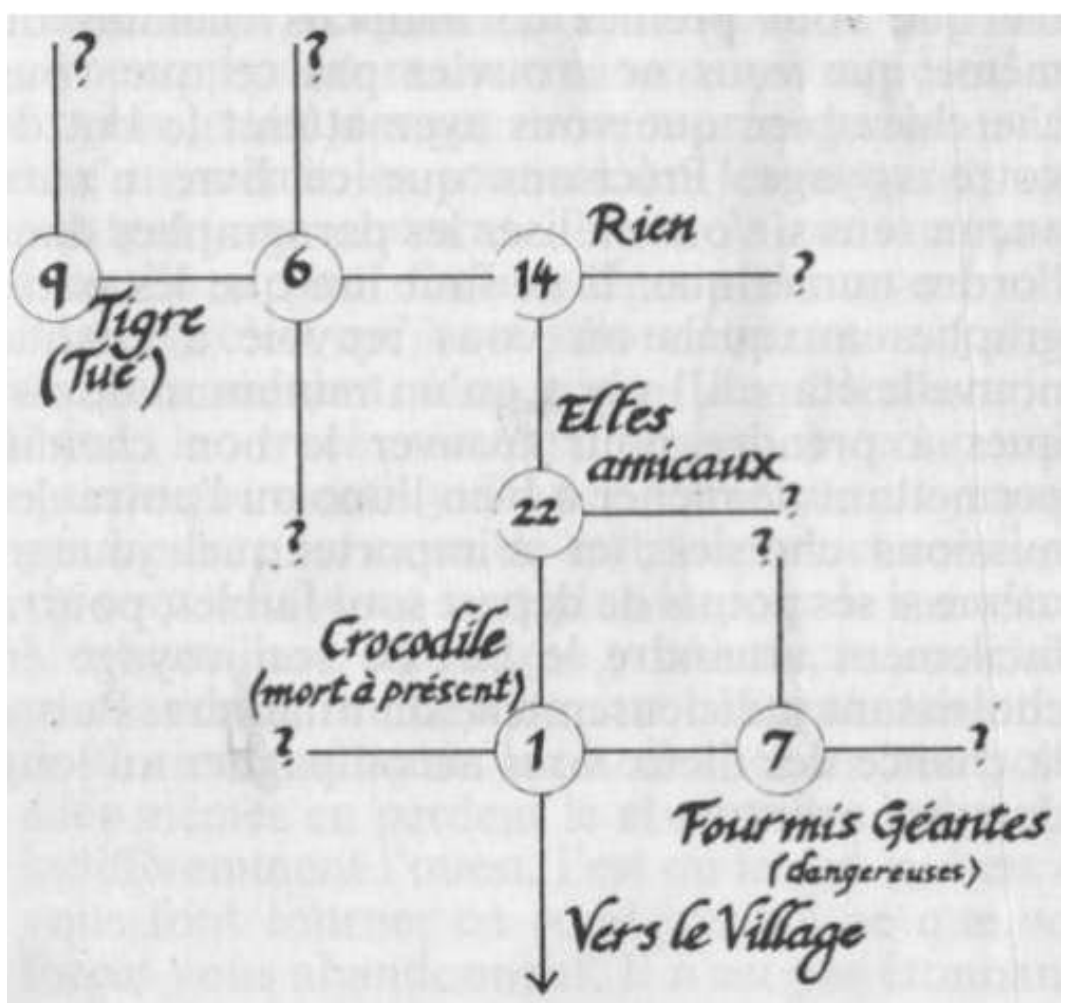

Un choix intéressé dans Le Marais aux scorpions (Jackson, 1984, illustration de Duncan Smith)

Ainsi, Le marais au Scorpions (Jackson, 1985a, p. 23) utilise la carte faite par le joueur comme marqueur de ses efforts, pour matérialiser sa réussite: être le premier à accomplir l'exploit de cartographier le terrible marais.

\section{Expérience de lecture ou expérience ludique?}

Notre typologie montre que la difficulté - comme l'intérêt de choisir - est avant tout marquée par l'indétermination (choix incertain) qu'implique la notion même de choix : mettre fin à l'un des termes équilibrés (choix équitable) d'une alternative afin d'en réaliser l'autre (choix ambivalent), d'où le risque de faire le mauvais choix (choix risqué) en se fermant à jamais (choix irrémédiable) les possibilités ouvertes par le choix précédent, et la tentation légitime de prolonger l'indécision jusqu'à être contraint (choix inévitable) ou motivé (choix intéressé) à se décider afin de l'emporter... ou de tout perdre (choix décisif). Ce dénouement de l'alternative est alors à même d'en ouvrir de nouvelles, entre lesquelles le joueur devra à nouveau se déterminer. Cette circularité conduit le joueur à affiner sa stratégie au fur et à mesure des parties, en fonction de ses essais-erreurs. Les auteurs du Sorcier de la Montagne de feu en avertissent leur lecteur en introduction de l'ouvrage :

Vous trouverez diverses clés dans les salles du souterrain. Mais vous ne parviendrez à vous emparez du trésor qu'en ayant avec vous la clé ouvrant le coffre qui le contient. Il faudra vous attendre à de nombreuses déceptions lorsque vous serez dans la montagne au sommet de feu. (Jackson \& Livingstone, 1984, p. 22-23)

A l'inverse, l'expérience de lecture est linéaire, puisque le fait de tourner les pages est sous-tendu par l'attente de ce qui va survenir. Dès lors l'obligation de recommencer ne 
peut apparaître que comme fastidieuse, ce dont témoigne le texte même du Sorcier de la Montagne de feu :

Si vous n'avez pas les trois clefs numérotées, voici venue la fin de votre aventure. Vous n'avez plus qu'à vous asseoir sur le coffre et à pleurer, car vous venez de comprendre qu'il va falloir recommencer à explorer la montagne depuis le début pour trouver les clefs qui vous manquent. (Jackson \& Livingstone, 1984, \$139)

Cette prise de conscience n'a cependant pas empêché les auteurs de prévoir la possibilité d'échouer dès la $7^{\mathrm{e}}$ alternative, dans la mesure où il est possible de rater l'une des clefs indispensables à la réussite de l'aventure, alors même que le joueur ne s'en rendra compte que trois paragraphes avant la fin d'une aventure qui en compte au minimum 110. Pour profiter du jeu sans nuire à l'intérêt littéraire, la stratégie utilisée par le lecteur incarne alors généralement une voie moyenne qui, entre astuce et tricherie, consiste à garder un doigt entre les dernières pages consultées pour marquer le paragraphe d'où il vient, au cas où l'un de ses choix l'obligerait à tout recommencer.

Bien entendu, cette "sauvegarde manuelle ", si elle est favorable à lecture, est à même de contrarier le plaisir suscité par la réussite de l'aventure. En effet, le plaisir de l'emporter tient au respect des règles qui fixent la difficulté. Or abaisser le niveau de celle-ci ne peut que diminuer d'autant la satisfaction d'avoir réussi. Cette difficulté est vantée par des titres qui en exagèrent les périls - La citadelle du chaos, La forêt de la malédiction, La galaxie tragique, Le labyrinthe de la mort, Le manoir de l'enfer... - afin de donner une dimension épique à la quête et accroître le mérite de la réussite. A l'inverse, l'approche narrative de "Choisis ta propre aventure " (titre français pour The adventure of you) en exalte le mystère : L'île canne-à-sucre emprunte à Defoe, Ton nom de code est Jonas à Fleming, La grotte du temps à Wells, Voyage sous les mers à Verne... mystère qui doit susciter l'envie de découverte. L'idée de contradiction entre jeu et narration a été particulièrement entretenue par les jeux vidéo, où lorsqu'une scène cinématique survient elle interrompt le jeu. Pourtant, un joueur est tour à tour acteur et spectateur de son jeu, et « une suite de choix intéressants » ne le serait plus si ceux-ci n'avaient pas d'effet sur l'engagement et l'immersion du joueur. La leçon des livres-jeux sur ce point est sans doute que ses exemples les plus réussis ont éveillé à la fois l'intérêt du lecteur et du joueur en mêlant adroitement les deux motivations que sont la découverte (attente) et le défi (risque), mais surtout en mettant le jeu au service de la fiction et inversement, c'est-à-dire en améliorant leur cohérence.

\section{Conclusion}

Ainsi, les fonctions constitutives du ludique ne s'apprécient pas en terme de comparaison mais de cohérence. Questionner la marge est précieux car, à l'inverse de l'opération formelle qui consiste à définir le jeu et qui n'a de regard que pour son centre, les confins du jeu, en nous faisant assister à la disparition du ludique, sont susceptibles de nous révéler ses fonctions à l'instant où nous prenons conscience que sa magie n'opère plus. La marge est ainsi moins l'expression d'une frontière formelle que celle d'une hybridation complexe (en quoi le jeu agence-t-il de manière singulière les éléments qu'il partage avec d'autres médiums comme la littérature ?) qui voit le jeu, qui dictait jusqu'alors ses règles à la narration, se fondre dans le roman avec lequel il partage bien un objectif commun - la satisfaction du désir de divertissement du lecteur - mais par un parcours inverse. Les potentialités, ces fonctions de l'indétermination 
ludique, permettent ainsi au joueur de s'irréaliser (au sens où il s'agit seulement d'une expérience fictive) à travers elles en devenant co-créateur de son expérience, alors que la lecture est à l'inverse une expérience d'abandon et de décorporation qui donne une profondeur aux choix ludiques en les mettant en perspective.

\section{BIBLIOGRAPHIE}

BARONI R. (2007), La tension narrative : suspense, curiosité et surprise, Paris, Seuil.

BORGES J. L. (1983 [1941]), Le Jardin aux sentiers qui bifurquent, in Fictions, Paris, Gallimard.

BOSS G. (2006 [1979]), Jeu et philosophie in Explorations et inventions : I. Lieux philosophiques, Québec, Editions du Grand Midi.

CAILLOIS R. (1958), Les jeux et les hommes : le masque et le vertige, Paris, Gallimard.

DEVER J. (1985 [1984]), La traversée infernale, Paris, Gallimard.

DUFLO C. (1997), Jouer et philosopher, Paris, Presses universitaires de France.

FREYHEIT M. (2014), Un coup de dé jamais n'abolira la lecture! Les livres dont vous êtes le héros et la matérialisation de lire, Université Paris 13, colloque La littérature de jeunesse dans le jeu des cultures matérielles et médiatiques : circulations, adaptations, mutations, 24-26 septembre 2014.

GOFFMAN E. (1974 [1979]), Les rites d'interaction, Paris, Minuit.

GOFFMAN E. (1991 [1974]), Les cadres de l'expérience, Paris, Minuit.

GYGAX G. \& ARNESON D. (1974), Dungeons and dragons, Lake Geneva, TSR.

HENRIOT J. (1983), Le jeu, Paris, Synonyme.

HUIZINGA J. (1951 [1938]), Homo ludens, Paris, Gallimard.

JACKSON S. (1984a [1983]), La citadelle du chaos, Paris, Gallimard.

JACKSON S. (1984b [1983]), La galaxie tragique, Paris, Gallimard.

JACKSON S. (1985a [1984]), Le marais aux scorpions, Paris, Gallimard.

JACKSON S. (1985b [1984]), Le manoir de l'enfer, Paris, Gallimard.

JACKSON S. \& LIVINGSTONE I. (1982), The Warlock of Firetop Mountain, Londres, Puffin Books.

JACKSON S. \& LIVINGSTONE I., (1984 [1983]), Le sorcier de la montagne de feu, Paris, Gallimard.

LIVINGSTONE I. (1984a [1983]), La forêt de la malédiction, Paris, Gallimard.

LIVINGSTONE I. (1984b), Le labyrinthe de la mort, Paris, Gallimard.

MONTGOMERY R. A. (1983 [1979]), Voyage sous les mers, Paris, Editions du Pélican.

PEREC G. (2010 [1968]), L'art et la manière d'aborder son chef de service pour lui demander une augmentation, Paris, Seuil.

PACKARD E. (1978 [1976]), Sugarcane Island, New York, Pocket books. 
PACKARD E. (1981a [1979]), La grotte du temps, Paris, Editions du Pélican.

PACKARD E. (1981b [1979]), Ton nom de code est Jonas, Paris, Editions du Pélican.

PICARD M. (1986), La lecture comme jeu : essai sur la littérature, Paris, Les éditions de Minuit.

PROPP V. (1970), Morphologie du conte (1928), Paris, Seuil.

QUENEAU R. (1982), Un conte à votre façon (1967), Paris, Kickshaws.

RYAN M.-L. (2007), « Jeux narratifs, fictions ludiques », Intermédialités, 9, pp. 15-34, https:// www.erudit.org/revue/im/2007/v/n9/1005527ar.html.

REY A. (dir.) (1994), Dictionnaire historique de la langue française, Paris, Le Robert.

ROLLINGS A. \& Morris D. (1999), Game architecture and design, Londres, New Riders Games.

SOLINSKI B. (2015), Ludologie : jeu, discours, complexité, Thèse de doctorat en sciences de l'information et de la communication, Metz, Université de Lorraine.

\section{NOTES}

1. La série de "Livres dont vous êtes le héros » Sorcellerie! permet ainsi de lancer les dés en feuilletant simplement les pages du livre au bas desquelles sont imprimées des faces de dés, et la série Loup solitaire grâce à une "table de hasard ", autrement dit un tableau numérique en fin d'ouvrage dont on désigne au hasard un chiffre en faisant glisser son crayon.

2. Par l'intermédiaire de Chainmail, jeu de figurines de Jeff Perren et Gary Gygax, publié chez Guidon games en 1971, qui se dota de règles spécifiques aux univers fantastiques.

3. "Il s'agit de livres dans lesquels on pose par exemple une question à la page 25 , l'élève y répond puis compare sa réponse aux réponses inscrites dans le livre (sous un cache). Cet enseignement programmé repose initialement sur une structure linéaire, mais évolue rapidement vers une structure ramifiée (Crowder, 1960) : par exemple, selon qu'il réponde a, b ou c, l'apprenant sera invité à se rendre à la page 3, 22 ou 44. Il sera donc confronté à diverses activités selon ses réponses. Il s'agit des premières solutions techniques au besoin d'individualisation.» (http://edutechwiki.unige.ch/fr/Enseignement_programmé_consulté le 31 mai 2016).

4. Il s'agit de la traduction populaire du terme « hack \& slash» employé dans les années 80 pour qualifier ce genre de jeux de rôle.

\section{RÉSUMÉS}

Les marges du jeu questionnent le moment et la façon dont le ludique devient autre. Dans le livre-jeu ces marges permettent de sonder l'articulation entre lecture et ludique et leurs fonctions respectives. L'histoire de l'émergence des livres à jouer permet d'analyser par comparaison l'apport des Livres dont vous êtes le héros à la dimension ludique du genre, d'où émerge celle de l'alternative et de la décision qui tranche cette alternative, permettant à partir des arbres de possibilités que dessine la lecture de ces ouvrages de dresser une typologie possible des choix ludiques. L'étude des contraintes que ceux-ci imposent et des potentialités qu'ils 
ouvrent, tant du point de vue du texte que de son usage, est alors à même de témoigner de la fécondation réciproque des approches narratives et ludiques.

The margins of the game question the moment and the way ludic becomes something else. In the book-game these margins allow to probe the articulation between reading and play and their respective functions. The literary roots of readings-to-play allow to analyse the contribution of Fighting Fantasy Gamebooks to ludic dimension of the genre, where emerges alternative and the decision that slices it. The reading of these books elaborates trees of opportunities that draw up a possible typology of playful choices. The study of the constraints and opportunities they open, from the point of view of the text and its use, demonstrates the cross-fertilization of narrative and ludic approaches.

\section{INDEX}

Keywords : choice, play, reading, ludics, ludology, narrative, potentiality, semiotics Mots-clés : choix, jeu, lecture, ludique, ludologie, narration, potentialité, sémiotique

\section{AUTEUR}

\section{BORIS SOLINSKI}

Université de Lorraine 